\title{
Pulmonary Hypertension Associated with HIV Infection on Nine Years Old Child
}

\author{
Baskoro Adi Prabowo*, Abdus Samik Wahab, Hasanah Mumpuni \\ Department of Cardiology and Vascular Medicine, Faculty of Medicine, Universitas Gadjah Mada - \\ Dr. Sardjito Hospital, Yogyakarta. Indonesia \\ *correspondence: baskoro27@gmail.com
}

\begin{abstract}
Introduction: Pulmonary arterial hypertension $(\mathrm{PAH})$ is an serious lifethreatening and severe complication of HIV infection. PAH Presentation in patient in HIV tend to non specific, resulted in recognized diagnosis at a later stage. Pulmonary Hypertension Associated with HIV Infection (PH-HIV) has been documented to occur at all stages of the disease and its manifestations range from asymptomatic right ventricular dysfunction to overt right heart failure. We presented this case to highlight the cardiovascular complication of HIV.
\end{abstract}

\section{Case report}

Case: A 9 years old child came to pediatric clinic with chief complaint a worsening dyspneu for 1 month, leg edema and difficulty lying on a flat bed. Patient was already diagnosed as HIV infection since three years old and regularly take HAART. At physical examination patient look dyspneic, heart rate 120 bpm, respiratory rate 40 times per minute, peripheral oxygen saturation $58 \%$ on room air, cyanotic lips and fingers, leg edema, chest examination showed RV heaving, with no cardiac murmur, rales heard at both lung, abdomen examination showed hepatomegaly and ascites. ECG findings sinus rythm, right axis deviation, right ventricular hypertrophy. Echocardiography findings showed right ventricular and atrial enlargement, mild to moderate tricuspid regurgitation ( TVG $50 \mathrm{mmHg}$, TR velocity $3.6 \mathrm{~m} / \mathrm{s}$ ), mild pulmonary regurgitation, Ejection fraction normal, TAPSE 8, no cardiac shunts found. Blood examination showed CD 484 cell/ $\mu \mathrm{L}$. Patient treated with furosemid $20 \mathrm{mg} / 8$ hours, spironolacton 12.5 $\mathrm{mg} / 24$ hour, lisinopril $2 \mathrm{mg} / 12$ hours, sidenafil $7 \mathrm{mg} / 8$ hours, and HAART duviral 1/2 tab/12 hours, aluvia 1 tab/12 hours. Patient treated at pediatric ward for 3 weeks and discharged in relieved condition and symptoms.

Discussion: PH-HIV histopathologic characteristics are not different from those idiopathic $\mathrm{PH}$. Pulmonary vascilature is obliterated with medial hypertrophy and increased proliferation of endothelial and smooth muscle cells. Plexiforms lesions can be detected in $78 \%$ patient with HIV PH. Whether there is no evidence that HIV was found infected lung vasculature but virus's protein and their interactions are strong candidate for cause-effect relationship between $\mathrm{PH}$ and HIV. HIV plays role by means of chronic inflamation and immune activation produced by HIV infection may lead increased secretion proinflamatory cytokines and growth factors that may promote PAH. Majority of the patients clinically present withdyspnoea on exertion (85\%), pedal oedema (20-30\%)and a non-productive cough (19\%). ECG finding that may support the diagnosis of $\mathrm{PH}$ is right axis deviation, $\mathrm{P}$ pulmonal, right ventricular hypertrophy, right bundle branch block, and prolonged QT interval. Echocardiography is the non invasive test of choice for initial screening for $\mathrm{PH}$ in symptomatic HIV patients. It is useful for identifying potential causes of $\mathrm{PH}$, evaluating RV function, and assessing related comorbidities. Treatment for HIV-PH use the same algorithm with $\mathrm{PAH}$ and should considered and taking considerations co-morbidities and drug interactions.

Conclusion: $\mathrm{PH}-\mathrm{HIV}$ is an important cardiovascular complication of HIV infection. The early initial diagnosis and treatment may improve functional class and symptoms.

Keywords: Pulmonary hypertension; HIV; child 